\title{
Prednisona e meloxicam no tratamento de ratos submetidos ao trauma agudo da medula espinhal
}

\author{
Prednisone and meloxicam in the treatment of rats underwent to acute spinal cord injury
}

\author{
Graciane Aiello ${ }^{I}$ Alexandre MazzantiI" Diego Vilibaldo Beckmann"II \\ Rosmarini Passos dos Santos ${ }^{\mathrm{I}}$ Amanda Oliveira de Andrades ${ }^{\mathrm{I}}$ \\ Heloisa Einloft Palma ${ }^{\mathrm{I}}$ Cinthia Melazzo de Andrade ${ }^{\mathrm{II}}$ André Vasconcelos Soares ${ }^{\text {II }}$
}

\section{RESUMO}

O objetivo deste estudo foi investigar o efeito da prednisona e do meloxicam na terapia de ratos submetidos ao modelo experimental de trauma agudo da medula espinhal, induzida pelo cateter de Fogarty $2 \mathrm{Fr}$, mediante a avaliação dos parâmetros de estresse oxidativo, dos testes neurológicos e do exame histopatológico da medula espinhal. Foram utilizados 90 ratos Wistar, distribuídos em seis grupos, denominados controle salina ou GCS (n=15), controle prednisona ou GCP $(n=15)$, controle meloxicam ou GCM $(n=15)$, trauma mais salina ou GTS (n=15), trauma mais prednisona ou GTP $(n=15)$ e trauma mais meloxicam GTM $(n=15)$. Cada grupo foi redistribuído em três subgrupos de igual número, de acordo com o tempo de tratamento no pós-operatório de $24 \mathrm{~h}, 72 \mathrm{~h}$ e sete dias. Todos os grupos foram submetidos à laminectomia e, nos grupos GTS, GTM e GTP, após a exposição da medula espinhal, foi realizado o trauma medular compressivo, utilizando o cateter de Fogarty $2 F r$. Os grupos GCS e GTS foram tratados com solução salina, os GSM e GTM receberam meloxicam e os GSP e GTP prednisona, sendo administrados pela via intraperitoneal. Em todos os ratos, foram avaliados os parâmetros de estresse oxidativo, testes neurológicos e exame histopatológico da medula espinhal. Os animais dos grupos GTS, GTM e GTP, nos diferentes tempos (24h, 72 h e sete dias), tiveram pontuação zero na escala de Basso, Beattie e Bresnahan (BBB); no plano inclinado, permaneceram com pontuação três e perderam a percepção da dor profunda. Os grupos GTM e GTP apresentaram menor atividade da catalase $e$ de níveis de TBARS, quando comparado ao grupo GTS. Foi constatada degeneração Walleriana e necrose da substância cinzenta de intensidades variáveis, não apresentando diferença entre os grupos submetidos ao trauma. O meloxicam e a prednisona apresentam possível efeito antioxidante, mas não impedem a necrose e a degeneração Walleriana da medula espinhal de ratos.

Palavras-chave: peroxidação lipídica, catalase, neurocirurgia.

\section{ABSTRACT}

The aim of the study was investigate the use of the prednisone and meloxicam in treatment of rats underwent to the experimental model of acute spinal cord injury with $2 \mathrm{Fr}$ Fogarty catheter, with evaluation of the oxidative stress, neurological test and histopathological analysis of the spinal cord. Ninety rats were separated into six equal groups denominated saline control or SCG, prednisone control or PCG, meloxicam control or MCG, saline and injury or STG, prednisone and injury PTG and meloxicam and injury MTG. Each group was divide into three subgroups according to treatment time in the postoperative period of $24 \mathrm{~h}, 72 \mathrm{~h}$ and seven days. All the rats underwent laminectomy and in the groups $S T G, M T G$ and PTG, after exposure of the spinal cord it was performed a compressive spinal cord injury with a $2 \mathrm{Fr}$ Fogarty catheter. The SCG and STG were treated with saline, MSG and MTG, with meloxicam and PSG and PTG with prednisone. All rats were evaluated for oxidative stress, neurological tests and histopathology of the spinal cord. Neurological tests were performed with Basso, Beattie e Bresnahan score (BBB), inclined plane and deep pain 24 hours before and after surgery and repeated every 48 hours until the day of euthanasia. The groups $S T G, M T G$ and PTG in the different times were zero point in the $B B B$ scale and three points in the inclined plane and absence of deep pain. MTG and PTG had lower catalase activity and TBARS levels when compared to the STG. In the histopathological analysis it was found Wallerian degeneration and necrosis of gray matter of intensity variation. Meloxicam and prednisone can exhibit antioxidant effect, but the necrosis and Wallerian degeneration were not stop in rats underwent to acute spinal cord injury.

Key words: lipid peroxidation, catalase, neurosurgery.

\section{INTRODUÇÃO}

A formação precoce de espécies reativas de oxigênio (EROs), também conhecidas como radicais

'Programa de Pós-graduação em Medicina Veterinária, Universidade Federal de Santa Maria (UFSM), Santa Maria, RS, Brasil.

"IDepartamento de Clínica de Pequenos Animais (DCPA), Centro de Ciências Rurais (CCR), UFSM, Avenida Roraima, 1000, 97105-900,

Santa Maria, RS, Brasil. E-mail: alexamazza@yahoo.com.br. *Autor para correspondência.

IIIniversidade Federal do Pampa (UNIPAMPA), Uruguaiana, RS, Brasil. 
livres tem sido implicada como a maior causa de degeneração do sistema nervoso central (SNC) após o trauma (HALL, 2011), gerando um quadro de estresse oxidativo. O mais conhecido efeito deste estresse é a oxidação em todos os componentes celulares, principalmente os ácidos graxos insaturados da membrana, levando à peroxidação lipídica (BRAUND et al., 1990). Os radicais livres são neutralizados por um sistema antioxidante enzimático (catalase e superóxido dismutase) e não enzimático (vitaminas $\mathrm{C}$ e E), promovendo assim a proteção das membranas biológicas (SCHMATZ et al., 2012).

Como o trauma primário não pode ser evitado e tampouco amenizado com tratamento farmacológico, os mecanismos secundários têm sido alvos de tais terapias. Cada agente terapêutico atua sobre um ou mais mecanismos da lesão secundária, objetivando conferir neuroproteção e/ou restauração do tecido lesado (FEHLINGS \& BAPTISTE, 2005).

Os anti-inflamatórios esteroidais, representados pelos glicocorticoides, têm sido exaustivamente pesquisados em trauma medular por suas diversas ações moduladoras sobre os mecanismos secundários da lesão, porém, seus efeitos colaterais são frequentes (BRAUND et al., 1990;). Já, os anti-inflamatórios não esterioidais, como, por exemplo, o meloxicam, têm demostrado características neuroprotetoras relevantes ao reduzir o estresse oxidativo em ratos com trauma medular agudo e estão associadas a mínimos efeitos colaterais (HAKAN et al., 2011).

Considerando a relevância dos estudos destinados às lesões traumáticas da medula espinhal em humanos e animais, o objetivo deste estudo foi investigar o efeito da prednisona e meloxicam na terapia de ratos submetidos ao modelo experimental de trauma agudo da medula espinhal, induzida pelocateter de Fogarty $2 \mathrm{Fr}$, mediante avaliação dos parâmetros de estresse oxidativo no sangue, dos testes neurológicos e do exame histopatológico da medula espinhal.

\section{MATERIAL E MÉTODOS}

Foram utilizados 90 ratos Wistar, machos, com peso corporal variando entre 290 e $360 \mathrm{~g}$, provenientes do Biotério Central da Instituição e distribuídos aleatoriamente em seis grupos de igual número $(\mathrm{n}=15)$, denominados de GCS ou controle salina, GCP ou controle prednisona, GCM ou controle meloxicam, GTS ou trauma mais salina, GTP ou trauma mais prednisona e GTM ou trauma mais meloxicam. Cada grupo foi redistribuído em três subgrupos $(n=5)$, de acordo com os tempos de avaliações pós-operatório, ou seja, 24h, 72h e sete dias, visto serem os períodos de maiores evidências das lesões secundárias ao trauma agudo de medula espinhal (PROFYRIS et al., 2004). Os ratos receberam ração comercial para roedores e água $a d$ libitum e foram mantidos em temperatura ambiente controlada $\left(21^{\circ} \pm 2^{\circ} \mathrm{C}\right)$ e com ciclos de 12 horas com luz e 12 horas sem luz.

Para a realização do procedimento cirúrgico, os animais foram submetidos à medicação pré-anestésica com cloridrato de tramadol, na dose de $10 \mathrm{mgkg}^{-1}$, por via subcutânea (SC) e indução e manutenção anestésica com isofluorano, administrado por meio de máscara em sistema semi-aberto. Após a realização da tricotomia e em plano anestésico, os ratos foram posicionados em decúbito ventral e feita antissepsia da pele com álcool-iodo-álcool.O acesso à medula espinhal foi realizado pela técnica de laminectomia dorsal em L3, conforme técnica descrita por SILVA et al. (2008). Em seguida, nos ratos dos grupos GCS, GCM e GCP, foi realizada a síntese da musculatura epaxial, com padrão de sutura simples contínuo e da pele com Wolff, ambos com fio de mononailon 4-0. Nos ratos dos grupos GTS, GTM e GTP, após a exposição da medula espinhal, foi introduzido o cateter de Fogarty $^{l} 2 \mathrm{Fr}$ no espaço epidural, posicionado na face dorsal da medula espinhal e conduzido cranialmente, conforme técnica descrita por VANICKÝ et al. (2001) até o espaço intervertebral T12 e T13. Em seguida, o balonete do cateter foi inflado, injetando-se $0,20 \mathrm{ml}$ de ar ambiente, com o auxílio de uma seringa, permanecendo inflado durante cinco minutos. A síntese da musculatura epaxial e da pele foi realizada utilizando o mesmo padrão de sutura e o tipo de fio anteriormente descrito para os grupos GCS, GCP e GCM.Os ratos de todos os grupos receberam analgesia pós-operatória com cloridrato de tramadol (10 $\left.\mathrm{mgkg}^{-1}, \mathrm{SC}\right)$ a cada oito horas, durante 24 horas.

Os ratos dos grupos GCS e GTS foram tratados com solução salina a $0,9 \%$, na dose de $0,5 \mathrm{ml} /$ rato, intraperitoneal (IP), a cada 24 horas, GCP e GTP com prednisona ${ }^{2}$ dose de $2 \mathrm{mgkg}^{-1}$, IP, a cada 24 horas e GCM e GTM com meloxicam ${ }^{2}$ na dose de $2 \mathrm{mgkg}^{-1}$, IP, a cada 24 horas. A administração dos fármacos iniciou 60 minutos após o término do procedimento cirúrgico nos ratos de todos os grupos. A duração do tratamento variou de acordo com os subgrupos, sendo os de $24 \mathrm{~h}$ de pós-operatório (PO) tratados com uma aplicação das soluções correspondentes (salina, meloxican ou prednisona) e submetidos à eutanásia 24 horas da cirurgia; os de 72 horas de PO, tratados com três aplicações e realizada a eutanásia após 72 horas; e 
os subgrupos de sete dias de PO, tratados com sete aplicações e feita a eutanásia após sete dias de PO.

Os testes neurológicos foram realizados em todos os animais através da escala de Basso, Beattie e Bresnahan (BBB), do plano inclinado e da percepção à dor profunda. A escala BBB observa os movimentos da articulação do quadril, joelho, tornozelo e posição do tronco, cauda e patas traseiras. A partir destas observações, foram atribuídos pontos de zero a 21 , sendo zero o correspondente à ausência total de movimentos e 21 à presença de movimentos normais (BASSO et al., 1995). No teste do plano inclinado, os animais foram colocados individualmente em um plano inclinado, emborrachado, ajustável em diferentes ângulos $\left(10^{\circ}\right.$ até $\left.90^{\circ}\right)$. $\mathrm{O}$ ângulo foi gradualmente aumentado de zero até o ângulo que o animal não conseguia mais se manter na posição inicial por cinco segundos (RIVLIN \& TATOR, 1979). Utilizou-se uma escala de 1 a 4, na qual: 1 - o animal permaneceu em um ângulo de $30^{\circ}$, menor do que antes da cirurgia; e 4 - permaneceu no mesmo ângulo antes do procedimento cirúrgico. A percepção à dor profunda (PDP) foi mensurada pela compressão das falanges (periósteo) e da cauda, com o auxílio de uma pinça hemostática de Halsted. A PDP foi considerada ausente quando os animais não reagiram ao estímulo doloroso com vocalização e/ou olhar para o membro.Estas avaliações foram feitas 24 horas antes e depois ao procedimento cirúrgico, seguido de intervalos de 48 horas. Os testes de avaliação de percepção à dor profunda foi realizado 24 horas após o procedimento cirúrgico, apenas nos ratos dos grupos GTS, GTM e GTP.

Para avaliar os parametros do estresse oxidativo, os animais foram anestesiados em um recipiente contendo algodão embebido com isofluorano, realizada a coleta de sangue por punção cardíaca $(10 \mathrm{ml})$ e, após, foram submetidos a eutanásia, utilizando o mesmo agente anestésico. O conteúdo de substâncias reativas ao ácido tiobarbitúrico (TBARS) foi mensurado através de amostras de soro (JENTZSCH et al., 1996) e a atividade da catalase obtida do sangue total, segundo o método espectrofotométrico de NELSON \& KIESOW (1972).

Para análise histopatológica, os segmentos da medula espinhal de T11 a L1 foram fixados em solução de formol tamponado a $10 \%$, por um período mínimo de 72 horas, e encaminhados para processamento pela técnica de inclusão em parafina. Os fragmentos foram cortados longitudinalmente e sagitalmente, montados em lâminas e posteriormente coradas pela técnica de Hematoxilina e Eosina. As alterações consideradas durante a histopatologia foram a presença ou ausência de degeneração walleriana e necrose, sendo classificadas em leve, moderada ou acentuada.

Para análise estatítica da atividade da catalase e dos níveis de TBARS, aplicou-se análise de variância (ANOVA) de uma via, seguida de teste de comparações múltiplas de Duncan. Todos os resultados foram indicados pela média \pm do Erro Padrão da Média (EPM). A análise histopatológica foi feitapelo teste de comparações multiplas de Tukey e Wilcoxon Score, com nível de significância de 5\%.

\section{RESULTADOS E DISCUSSÃO}

Os testes neurológicos empregados neste estudo demonstraram que os ratos dos grupos GCS, GCM, GCP não apresentaram deficiências neurológicas nos diferentes tempos de observação. Este resultado corrobora o de KAPTANOGLU et al. (2003), que também não observaram tais alterações. Por outro lado, todos os ratos submetidos ao modelo experimental de trauma medular (GTS, GTM e GTP) e nos diferentes tempos ( $24 \mathrm{~h}, 72 \mathrm{~h}$ e sete dias) tiveram pontuação zero na escala $\mathrm{BBB}$, ou seja, nenhum movimento observado nos membros pélvicos, no plano inclinado, permaneceram com pontuação 3, ou seja, em um ângulo de dez graus menor que o anterior à cirurgia. Também perderam a percepção à dor profunda e, segundo BERGMAN et al. (2000), este pode ser um sinal clínico, cujo prognóstico é desfavorável em relação ao retorno da deambulação. Portanto, devido à grave lesão induzida na medula espinhal, justificada pela ausência da dor profunda, e ao curto período de observação pós-operatório, não era esperado observar recuperação funcional dos ratos submetidos ao trauma. A prednisona inibe ambas as vias da inflamação, a da cicloxigenase (COX-1 e COX-2) e da lipoxigenase e o meloxicam apresenta inibição seletiva da COX-2 (ENGELHARDT et al., 1996), devido a isso, o meloxicam ocasiona menor ocorrência de efeitos colaterais. Tem sido sugerido que, ao inibir a COX-2, não haverá a formação de fosfolipase A2, pois impede a liberação do ácido aracdônico da membrana fosfolipídica e assim não há a formação de eicosanoides e radicais livres (TAOKA \& OKAJIMA, 1998). Portanto, fármaco que inibe a COX-2 previne a lesão da membrana através da inibição da peroxidação e hidrólise de lipídios.

Para avaliar a formação de radicas livres, foi verificado, nesta pesquisa, o nível de TBARS. Este não apresentou diferença nos grupos controle (GCS, GCM e GCP) decorridas 24h e 72h de PO (Figura 1A). Já, aos sete dias de PO, os ratos dos grupos controle (GCS, 

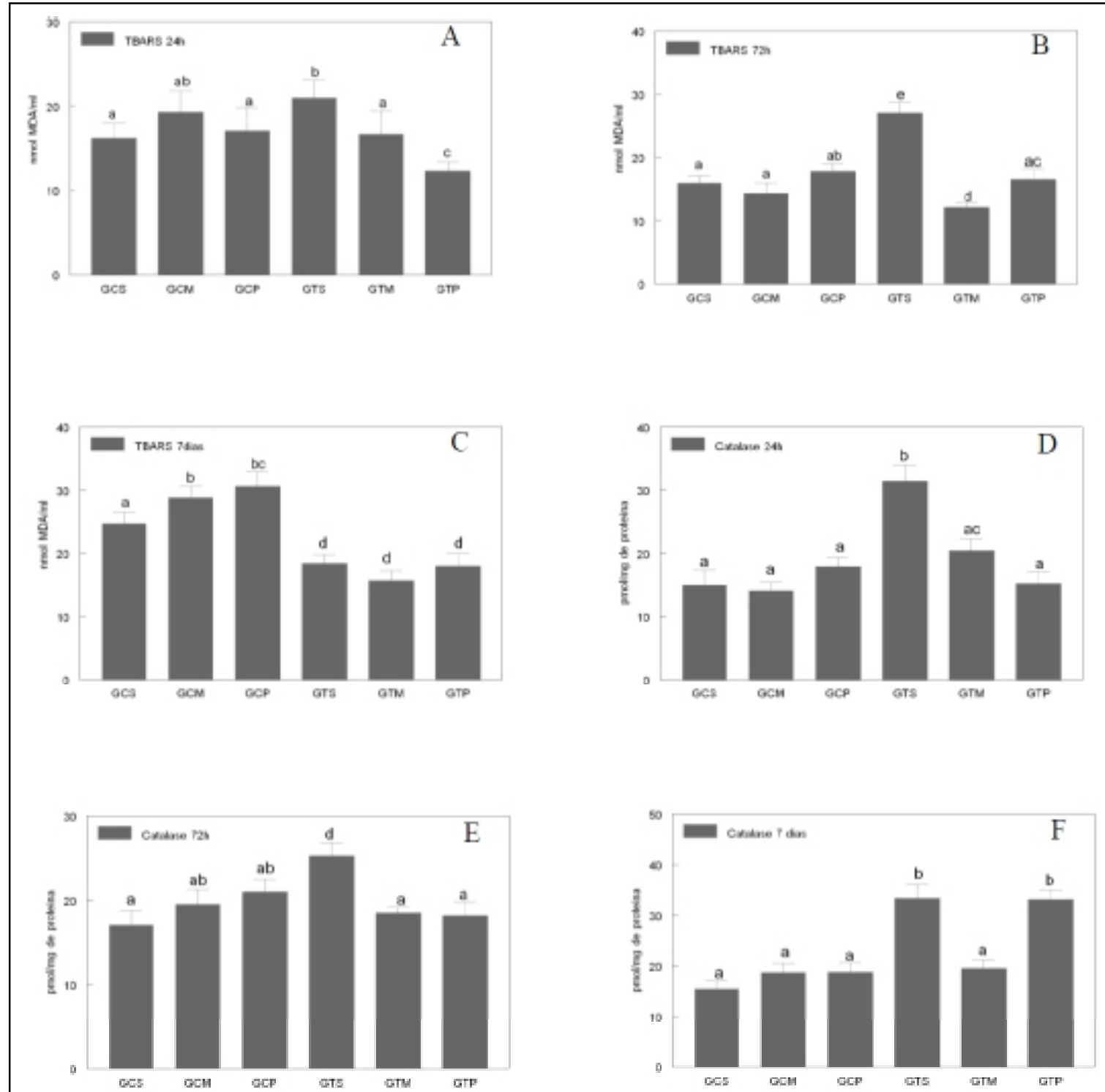

Figura 1 - Valores dos níveis de TBARS (A, B, C) e da atividade da catalase (D, E, F) de ratos submetidos a trauma agudo da medula espinhal, com cateter de Fogarty $2 F r$ e tratados com meloxicam ou prednisona, decorridas $24 \mathrm{~h}$, $72 \mathrm{~h}$ e sete dias de pós-operatório. GCS: Grupo controle salina; GCM: Grupo controle meloxican; GCP: Grupo controle prednisona, GTS: Grupo trauma salina; GTM: Grupo trauma meloxicam; GTP: Grupo trauma prednisona. Grupos com letras diferentes são estatisticamente diferentes $(\mathrm{P}<0,05 ; \mathrm{n}=5)$.

GCM e GCP) apresentaram o nível de TBARS maior, quando comparado aos demais tempos de avaliação pós-operatória, indicando que a peroxidação lípidica foi maior, mesmo sem os estímulos provocados pelo trauma. Ainda, neste mesmo período de avaliação, o GCS apresentou níveis de TBARS inferior $(\mathrm{P}<0,05)$ aos grupos GCM e GCP (Figura 1C). Nos grupos traumatizados, o nível de TBARS, após 24 horas de PO, nos grupos GTM e GTP, apresentou níveis inferiores ao GTS $(\mathrm{P}<0,05)$ (Figura 1A). Estes resultados demonstraram que o trauma medular realizado pelo cateter $\mathrm{Fogarty} 2 \mathrm{Fr}$ levou a um significante aumento na peroxidação lipídica. Quando os ratos foram tratados com anti-inflamatórios esteroidal ou não esteroidal, observou-se uma diminuição significativa na peroxidação lipídica, sugerindo uma ação antioxidante destes fármacos.

O grupo GTP também apresentou um nível de TBARS inferior ao grupo GTM $(\mathrm{P}<0,05)$, demonstrando que a prednisona mostrou-se um efeito antioxidante melhor neste período, ao ser comparado ao GTM. Decorridas 72 horas de PO, o grupo GTS também apresentou nível de TBARS maior que os demais grupos tratados $(\mathrm{P}<0,05)$, porém, ao contrário 
do resultado observado nas primeiras 24 horas de PO, no grupo GTM, o nível de peroxidação lipídica foi inferior ao grupo GTP $(\mathrm{P}<0,05)$ (Figura 1B), indicando a persistência da atividade antioxidante $\mathrm{e}$ inibição da formação de radicais livres, promovida pelo meloxicam. Aos sete dias de PO, no entanto, os níveis de peroxidação lipídica do GTS reduziram aos valores próximos aos dos grupos GTM e GTP, não havendo diferença entre eles. Pode-se sugerir que este fato está relacionado à ausência ou diminuição de substrato (lipídios) aos sete dias de PO, visto que, nos períodos de 24 e $72 \mathrm{~h}$, foi observado um aumento significativo $(\mathrm{P}<0,05)$ da peroxidação lipídica, provavelmente, pela disponibilidade ainda de material biológico (lipídios), devido ao trauma recente.

Em relação à atividade da catalase, não houve diferença nos grupos controle (GCS, GCM e GCP), decorridas $24 \mathrm{~h}$ e $72 \mathrm{~h}$ e sete dias de pósoperatório (PO). Este resultado demonstra que a atividade desta enzima foi semelhante nos grupos que não tiveram trauma medular e não receberam nenhum tipo de fármaco (Figuras 1D e 1E). No grupo submetido ao trauma medular (GTS), foi observado um aumento na atividade da catalase $(\mathrm{P}<0,05)$, uma importante enzima que atua na linha de frente, na tentativa de remover as EROs e consequentemente minimizar os danos causados por essas espécies reativas. Já nos grupos traumatizados e tratados (GTM e GTP), decorridas 24 e 72 horas de PO, verificouse uma diminuição na atividade da CAT, comparada com o GTS $(\mathrm{P}<0,05)$. Esses resultados indicam que o meloxicam e a prednisona apresentaram uma atividade antioxidante e impediram um aumento no estresse oxidativo. Aos sete dias de PO, no entanto, verificouse que o grupo GTM apresentou uma atividade da CAT inferior aos grupos GTS e GTP $(\mathrm{P}<0,05)$ e se igualou aos controles não traumatizados (Figura $1 \mathrm{~F}$ ), indicando que o meloxicam continuou por um tempo mais prolongado sua atividade antioxidante e possível ação neuroprotetora, corroborando os achados de STRAUSS \& MARINI (2002). HAKAN et al. (2011) também observaram, em ratos submetidos a trauma medular, que o tratamento com meloxicam apresentou características neuroprotetoras, ao determinar que este reduziu o estresse oxidativo pela da inibição da peroxidação lipídica e da fragmentação de DNA.

Nesta pesquisa, não foram observadas, no exame histopatológico do fragmento da medula espinhal dos ratos dos grupos controles, lesões na meninge e no parênquima medular em nenhum dos tempos avaliados (Figuras 2A e 2C). Este dado é similar aos de KAPTANOGLU et al. (2003), os quais também não observaram alterações microscópicas em ratos submetidos à laminectomia sem trauma

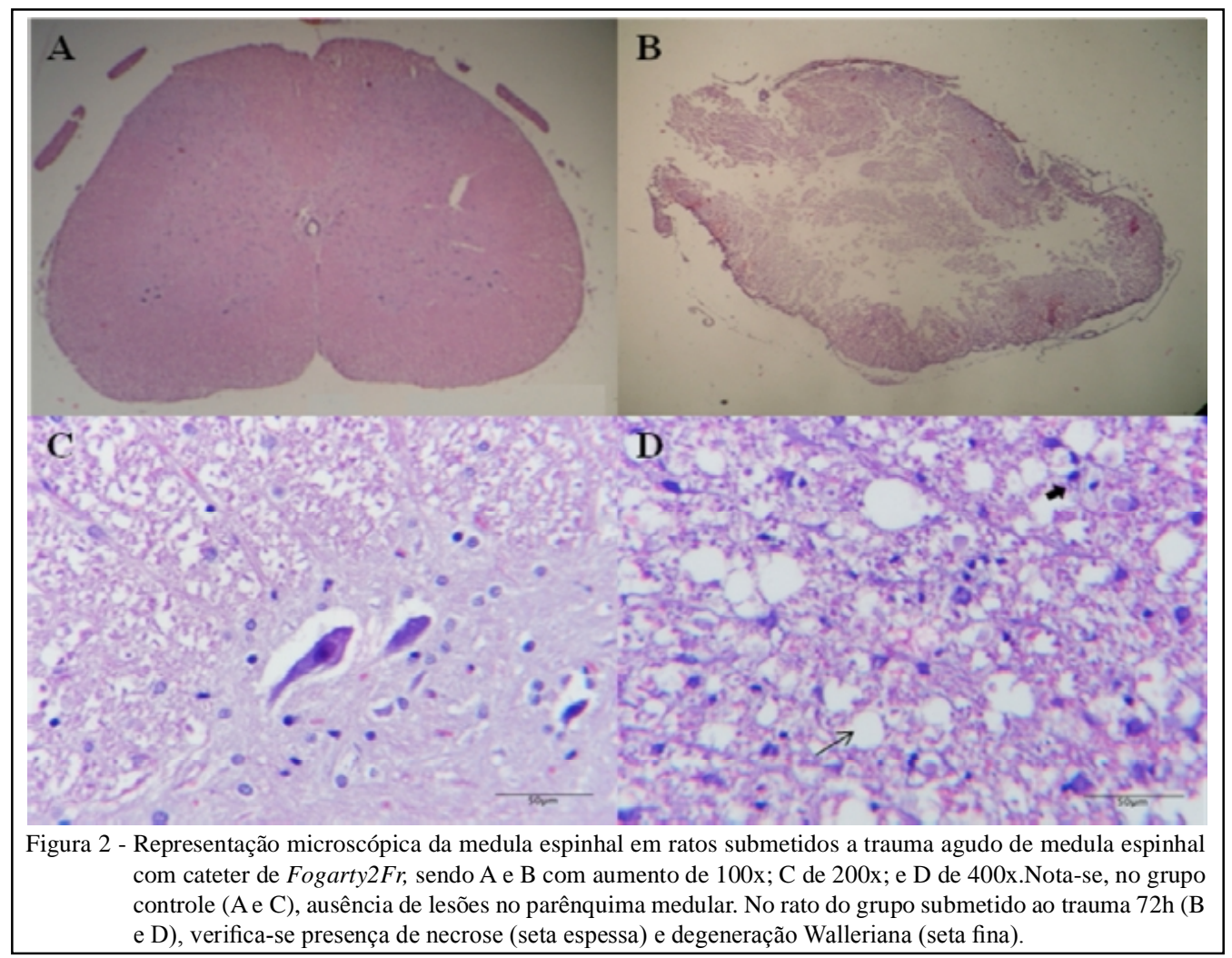

Ciência Rural, v.45, n.1, jan, 2015. 
medular, diferindo, porém, no período de avaliação, que foi até oito horas pós-trauma. Já nos ratos dos grupos submetidos ao trauma medular (GTS, GTM e GTP), foram observadas lesões de necrose da substância cinzenta e degeneração Walleriana de intensidades variáveis (Figuras $2 \mathrm{~B}$ e 2D) e em todos os tempos de observações ( $24 \mathrm{~h}, 72 \mathrm{~h}$ e sete dias). Estes resultados coincidem com os de CEMIL et al. (2010) e TOKLU et al. (2010), que verificaram estas mesmas alterações após o trauma medular agudo e com início geralmente após $24 \mathrm{~h}$ do trauma.

A degeneração Walleriana é o termo empregado para a degeneração de axônios e suas bainhas de mielina (substância branca) e geralmente é de origem traumática (CARLTON et al., 2009). Com relação a este achado, foi verificado, nos ratos do grupo GTS, 33,3\% de intensidade leve e $66,4 \%$ moderada e, no grupo GTM, $100 \%$ de intensidade moderada e no GTP $100 \%$ de intensidade acentuada após $24 \mathrm{~h}$ do trauma. Após $72 \mathrm{~h}$ de PO, foi observado, no grupo GTS, $66,4 \%$ de intensidade leve e $33,3 \%$ acentuada, no GTM, 33,3\% de intensidade moderada e $66,7 \%$ leve e, no GTP, $33,3 \%$ de intensidade leve, $33,3 \%$ moderada e 33,3\% ausência de degeneração Walleriana. Já nos ratos de sete dias de PO, o grupo GTS apresentou $66,7 \%$ de intensidade leve e $33,3 \%$ ausente, no GTM 33,3\% moderada e $66,7 \%$ acentuada e, no GTS $66,7 \%$ de intensidade acentuada e 33,3\% ausência de degeneração Walleriana. Não houve diferença entre os grupos nos tempos de observação PO, porém foi observada uma redução na intensidade da lesão, decorridas 72 horas de PO nos grupos GTM e GTP, quando comparado ao grupo GTS, que se manteve estável. HAKAN et al. (2011) também observaram que o grupo submetido ao trauma medular e tratado com meloxicam durante sete dias apresentaram degeneração menos intensa, comparado ao grupo tratado com solução salina. Já SILVA et al. (2008), em seu estudo, não observaram diferenças histopatológicas entre os grupos tratados com diferentes doses de prednisona ao grupo tratado com placebo.

Em relação à necrose da medula espinhal, foi verificado, nos ratos do grupo GTS, 33,33\% de intensidade leve e $66,4 \%$ moderada; no grupo GTM, $66,7 \%$ de intensidade leve e $33,3 \%$ moderada; e, no GTP, $100 \%$ de intensidade moderada após 24 horas de PO. Decorridas $72 \mathrm{~h}$ de PO, foi observado, no grupo GTS, 66,4\% de intensidade acentuada e 33,3\% moderada; no GTM, 33,3\% de intensidade leve e $66,7 \%$ acentuada; e, no GTP, $66,7 \%$ de intensidade leve e 33,3\% moderada. Já nos ratos de sete dias de PO, o grupo GTS apresentou $100 \%$ de intensidade acentuada; GTM 33,3\% moderada e 66,7\% acentuada; no GTP $66,7 \%$ de intensidade moderada e $33,3 \%$ acentuada. A necrose aumentou em todos os grupos de acordo com a evolução do PO. Não foi observada diferença entre os grupos GTS, GTM e GTP entre os tempos de observação, porém os grupos GTM e GTP apresentaram intensidade inferior ao grupo GTS.

Com relação às lesões microscópicas da medula espinhal, tanto o meloxicam como a prednisona não impediram a necrose e a degeneração Walleriana, porém a intensidade foi discretamente menor nos ratos tratados com estes fármacos, quando comparado ao grupo controle (salina). Embora se necessite de mais investigações a respeito, a relevância clínica deste estudo foi a possível influência no prognóstico, haja vista que a preservação de $5 \%$ dos tratos motores poderá recuperar a deambulação em ratos (BASSO et al., 1996). Novos estudos com maior amostragem de ratos são indicados para avaliaro efeito destes fármacos por um período prolongado de pós-operatório, estudar outros marcadores na medula espinhal como glutamato, acetilcolinesterases, quantificação dos tratos motores e sensoriais da medula espinhal e correlacioná-los com os sinais neurológicos.

Com os resultados encontrados neste estudo, pôde-se sugerir que o meloxicam e a prednisona apresentaram possível efeito antioxidante quando comparado ao grupo controle e foi mais evidente nas primeiras $72 \mathrm{~h}$ pós-trauma. $\mathrm{O}$ meloxicam possuiu uma ação mais prolongada que a prednisona, principalmente na diminuição da atividade da catalase, porém, nenhum deles apresentou vantagens na indicação no tratamento de ratos submetidos a trauma agudo de medula espinhal, empregando o cateter de Fogarty $2 \mathrm{Fr}$.

\section{CONCLUSÃO}

O modelo de trauma medular utilizado produz severas deficiências neurológicas. Os grupos tratados com prednisona e meloxicam sofreram uma possível ação antioxidante, devido aos níveis de peroxidação lipíca terem sido menores nestes grupos, pois estes fármacos apresentam provável ação antioxidante e neuroprotetora, sendo que a atividade antioxidande foi mais prolongada no grupo tratado com meloxicam.

\section{FONTES DE AQUISIÇÃO}

1. Edwards Lifesciences. São Paulo - SP.

2. Sigma - Aldrich. St Louis, MO - USA.

Ciência Rural, v.45, n.1, jan, 2015. 


\section{COMITÊ DE ÉTICA E BIOSSEGURANÇA}

Este trabalho foi aprovado pelo Comitê de Ética em Pesquisa da Universidade Federal de Santa Maria (UFSM) sob o número 001/2012.

\section{REFERÊNCIAS}

AMSELLEM, P.M. et al. Loss of Deep Pain Sensation Following Thoracolumbar Intervertebral Disk Herniation in Dogs: Treatment and Prognosis. Compendium on Continuing Education for the Practicing Veterinarian, v. 25, 266-273, 203. Disponível em: <http://www.vetlearn.com/compendium>.Acessoem:23 nov. 2011.

BASSO, D.M. et al. A sensitive and reliable locomotor rating scale for open field testing in rats. Journal of Neurotrauma, v.12, p.1-21, 1995. Disponível em: <http://online.liebertpub.com/ doi/pdf/10.1089/neu.1995.12.1>. Acesso em: 25 nov. 2011. doi: 10.1089/neu.1995.12.1.

BASSO M.D. et al. Graded histological and locomotor outcomes after spinal cord contusion using the NYU weight-drop device versus transection. Experimental Neurology, v.139, p.244-256, 1996. Disponível em: <http://www.sciencedirect.com/science/ article/pii/ S0014488696900989\#>. Acesso em: 20 nov. 2011. doi: 10.1006/exnr.1996.0098.

BRAUND, K.G. et al. The etiology, pathology, and pathophysiology of acute spinal cord trauma. Veterinary Medicine, v.85, p.684691,1990

CARLTON, S.M. et al. Peripheral and central sensitization in remote spinal cord regions contribute to central neuropathic pain after spinal cord injury. Pain, v.147, p.265-276, 2009. Disponível em: <http://www.sciencedirect.com/science/article/pii/ S0304395909005661>. Acesso em: 23 nov. 2011. doi: 10.1016/j. pain.2009.09.030.

CEMIL B. et al. Curcumin improves early functional results after experimental spinal cord injury. Acta Neurochirugica, v.152, n.9, p.1583-1590, 2010. Disponível em: <http://www.ncbi.nlm.nih. gov /pubmed/20535508>. Acesso em: 3 nov. 2011. doi: 10.1007/ s00701-010-0702-x.

ENGELHARDT, G. et al. Meloxicam: Influence on arachidonic acid metabolism. Part II. In vivo findings. Biochemistry and Pharmacology, v.51, p. 29-38, 1996. Disponível em: <http://www. ncbi.nlm.nih.gov/pubmed/8534265>. Acesso em: 23 nov. 2011. doi: 0.1016/0006-2952(95)02110-8.

FEHLINGS, M.G.; BAPTISTE, D.C. Current status of clinical trials for acute spinal cord injury. Injury, v.36, p.113-122, 2005. Disponível em: <http://www.sciencedirect.com/science/article/pii/ S0020138305002159>. Acesso em: 23 nov. 2011. doi: 10.1016/j. injury.2005.06.022.

HALL, E.D. Antioxidant therapies for acute spinal cord injury. American Society for Experimental Neuro Therapeutics, v.8, p.152-167, 2011. Disponível em: <http://www.ncbi.nlm.nih.gov /pmc/articles/PMC3101837/>. Acesso em: 23 nov. 2011. doi: 10.1007/s13311-011-0026-4.

HAKAN, T. et al. Meloxicam exerts neuroprotection on spinal cord trauma in rats. International Journal of Neuroscience, v.121, p.142-148, 2011. Disponível em: <http://informahealthcare.com/ doi/abs/10.3109/00207454.2010.537415>. Acesso em: 23 nov. 2011. doi: $10.3109 / 00207454.2010 .537415$.
JENTZSCH, A.M. et al. Improved analysis of malondialdehyde in human body fluids. Free Radical \& Medicine, v.20, n.2, p.251-256, 1996. Disponível em: <http://www.sciencedirect.com/ science/article/pii/0891584995020438 >. Acesso em: 23 nov. 2011. doi: 10.1016/0891-5849(95)02043.

KAPTANOGLU, E. et al. Effect of magnesium sulphate in experimental spinal cord injury: evaluation with ultrastructural findings and early clinical results. Journal of Clinical Neuroscience, v.10, n.3, p.329-334, 2003. Disponível em: <http:// www.sciencedirect.com/science/article/pii/S0967586803000316>. Acesso em: 20 nov. 2011. doi: 10.1016/S0967-5868(03)00031-6.

NELSON, D.P.; KIESOW, L.A. Enthalpy of decomposition of hydrogen peroxide by catalase at 25 degrees $\mathrm{C}$ (with molar extinction coefficients of $\mathrm{H}_{2} \mathrm{O}_{2}$ solutions in the UV). Analytical Biochemistry, v.49, n.2, p.474-478, 1972. Disponível em: <http:// www.ncbi.nlm.nih.gov/pubmed/5082943>. Acesso em: 13 nov. 2011. doi: 10.1016/0003-2697(72)90451-4.

PROFYRIS, C. et al. Degenerative and regenerative mechanisms governing spinal cord injury. Neurobiology of Disease, v.15, p.415-436, 2004. Disponível em: 〈http://www.sciencedirect.com/ science/article/pii/S0969996103002523>. Acesso em: 23 nov. 2011. doi: 10.1016/j.nbd.2003.11.015.

RIVLIN, A.S.; TATOR, C.H. Effect of vasodilators and myelotomy on recovery after acute spinal cord injury in rats. Journal of Neurosurgery, v.50, p.349-352, 1979. Disponível em: <http:// thejns.org/doi/abs/10.3171/jns.1979.50.3.0349\%40col.2012.116. issue-6? journalCode $=$ col $>$. Acesso em: 23 nov. 2011. doi: 10.3171/jns. 1979.50.3.0349.

SCHMATZ, R et al. Effects of resveratrol of oxidative stress and on the activity of delta aminolevulinic acid dehydratase in liver and kidney streptozotocin-induced diabetic rats. Biochimie, v.94, p.374-383, 2012. Disponível em: <http://www.sciencedirect.com/ science/article/pii/S0300908411003105>. Acesso em: 23 nov. 2011. doi: 10.1016/j.biochi.2011.08.005.

SILVA, C.M.O. et al. Efeito da prednisona em lesão medular aguda experimental em ratos. Arquivo Brasileiro de Medicina Veterinária e Zootecnia, v.60, n.3, p.641-650, 2008. Disponível em: <http://www.scielo.br/scielo.php?pid=S010209352008000300018\&script=sciarttext $>$. Acesso em: 20 nov. 2011. doi: 10.1590/S0102-09352008000300018.

STRAUSS, K.I.; MARINI, A.M. Cyclooxygenase-2 inhibition protects cultured cerebellar granule neurons from glutamatemediated cell death. Journal of Neurotrauma, v.19, p.627-638, 2002. Disponível em: <http://online.liebertpub.com/doi/abs/10 1089/089771502753754091>. Acesso em: 23 nov. 2011. doi: 10.1590/S0102-09352008000300018.

TAOKA, Y.; OKAJIMA, K. Spinal cord injury in the rat. Progress in Neurobiology, v.56, p.341-358, 1998. Disponível em: <http:// www.sciencedirect.com/science/article/pii/S0301008298000495>. Acesso em: 23 nov. 2011. doi: 10.1016/S0301-0082(98)00049-5.

TOKLU, H.Z. et al. Neuroprotective effects of alpha-lipoic acid in experimental spinal cord injury in rats. Journal of Spinal Cord Medicine, v.33, n.4, p.401-409, 2010. Disponível em: <http://www. ncbi.nlm.nih.gov/pmc/articles/PMC2964029/pdf/i1079-0268-334-401.pdf>. Acesso em: 23 nov. 2011. doi: 1079-0268-33-4-401.

VANICKÝ, I. et al. A simple and reproducible model of spinal cord injury induced by epidural balloon inflation in the rat. Journal of Neurotrauma,v.8,n.12,p.1399-1407,2001.Disponível em: <http:// online.liebertpub.com/doi/abs/10.1089/08977150152725687>. Acesso em: 23 nov. 2011. doi: 10.1089/08977150152725687. 